\title{
Impaired Recognition Memory in Monkeys after Damage Limited to the Hippocampal Region
}

\author{
Stuart M. Zola, ${ }^{1,2,3}$ Larry R. Squire, ${ }^{1,2,3,4}$ Edmond Teng, ${ }^{3}$ Lisa Stefanacci, ${ }^{2}$ Elizabeth A. Buffalo, ${ }^{3}$ \\ and Robert E. Clark ${ }^{2}$ \\ ${ }^{1}$ Veterans Affairs Medical Center, San Diego, California 92126, and ${ }^{2}$ Departments of Psychiatry, ${ }^{3}$ Neurosciences, and \\ ${ }^{4}$ Psychology, University of California, La Jolla, California 92093
}

\begin{abstract}
Monkeys with lesions limited to the hippocampal region (the hippocampus proper, the dentate gyrus, and the subiculum) were impaired on two tasks of recognition memory: delayed nonmatching to sample and the visual paired-comparison task. Recognition memory was impaired in five different groups of monkeys, whether the lesions were made by an ischemic procedure, by radio frequency, or by ibotenic acid. The finding that
\end{abstract}

the hippocampal region is essential for normal recognition memory performance is considered in the context of current ideas about the role of the hippocampus in declarative memory.

Key words: hippocampus; recognition memory; monkeys; radio-frequency lesions; ischemic lesions; ibotenic acid lesions; delayed nonmatching to sample; visual paired comparison
In mammals, the formation and storage of declarative memory depends on a system of anatomically related structures within the medial temporal lobe and on an interaction between this system and the neocortex (Squire, 1992a,b; Eichenbaum, 1997). The important structures within the medial temporal lobe are the hippocampal region (the hippocampal cell fields, the dentate gyrus, and the subiculum) and the adjacent entorhinal, perirhinal, and parahippocampal cortices (Zola-Morgan and Squire, 1993). Recently, interest has focused on the hippocampus. It has been suggested that although the hippocampus is important for many forms of memory, it may not be essential for the capacity to identify a recently encountered item as familiar, a capacity termed recognition memory (Eichenbaum et al., 1994; Aggleton and Shaw, 1996; Vargha-Khadem et al., 1997; Aggleton and Brown, 1999).

Information from studies with humans suggests that lesions restricted to the hippocampus do impair recognition memory. Thus, patients R.B. and G.D., who had ischemic damage limited to the CA1 field of the hippocampus and the CA1/subicular border zone (Zola-Morgan et al., 1986; Rempel-Clower et al., 1996), were impaired on various verbal and nonverbal memory tests, and recognition memory was impaired along with cued recall and free recall. Similar findings were obtained for patients with radiological evidence of damage restricted to the hippocampal formation (Reed and Squire, 1997; Manns and Squire, 1999). However, the data from humans are complicated by the possibility that ischemic damage might cause neuronal dysfunction (which does not progress to cell death) in structures beyond the hippocampus that are important for memory (Nunn and Hodges,

\footnotetext{
Received July 23, 1999; revised Sept. 7, 1999; accepted Oct. 7, 1999.

This research was supported by the Medical Research Service of the Department of Veterans Affairs, National Institutes of Health Grants MH58933, MH24600, MH11649, MH18399, and MH11154, and the McDonnell-Pew Center for Cognitive Neuroscience. We thank Cecelia Manzanares, Jeff Manzanares, Scott Hanson, Michelle Hu, Elaine Ellerton, and Cynthia Mills for excellent technical assistance.

Correspondence should be addressed to Dr. Stuart M. Zola, Department of Psychiatry, University of California School of Medicine, La Jolla, CA 92093. E-mail: szola@ucsd.edu.

Copyright (C) 1999 Society for Neuroscience $0270-6474 / 00 / 200451-13 \$ 15.00 / 0$
}

1994; Bachevalier and Meunier, 1996; Aggleton and Brown, 1999). Some experimental work speaks against the "hidden damage" hypothesis (Zola-Morgan et al., 1992; Squire and Zola-Morgan, 1996) but it is difficult to rule out this hypothesis entirely.

In the monkey, the assessment of recognition memory has depended mainly on two tasks. In delayed nonmatching to sample (Mishkin and Delacour, 1975), two objects are presented, a new one and one that was presented earlier, and the monkey must choose the new object. In the visual paired-comparison task, as adapted for the monkey (Bachevalier et al., 1993), a new picture and a recently presented picture are presented side by side, and the monkey's spontaneous tendency to look at the new picture is measured.

To date, four studies have assessed recognition memory in monkeys with lesions of the hippocampal region, all with the delayed nonmatching to sample task. The first two involved monkeys with ischemic (ISC) damage (Zola-Morgan et al., 1992) or monkeys with stereotaxic radio-frequency (RF) lesions (Alvarez et al., 1995). In both studies, damage limited to the hippocampal region impaired recognition memory.

More recently, selective neurotoxins, e.g., ibotenic acid (IBO), have been used to make fiber-sparing lesions limited to the hippocampal region in monkeys (Murray and Mishkin, 1998; Zola et al., 1998; Beason-Held et al., 1999). Ibotenic acid selectively damages cell bodies within the target region, although it spares adjacent white matter (Jarrard, 1989). Thus, lesions made by ibotenic acid should provide the best means of assessing the role of the hippocampal region in recognition memory. To date, only two studies have assessed recognition memory after ibotenic acid lesions directed at the hippocampal region. Consistent with findings from the studies that used ISC or RF lesions, BeasonHeld et al. (1999) reported impaired performance on two different tests of recognition memory: delayed nonmatching to sample and a delayed recognition span task. The other study (Murray and Mishkin, 1998) obtained different results. Monkeys with conjoint IBO lesions of the hippocampal region and the amygdala performed as well as unoperated monkeys on the delayed nonmatch- 
ing task. Accordingly, the available data from monkeys are not in agreement.

The present report attempts to clarify the nature of recognition memory and the role of the hippocampus. We present data for five groups of monkeys who sustained bilateral lesions limited to the hippocampal region (ISC, RF1, RF2, IBO1, and IBO2). Findings from the ISC and the RF1 groups have appeared previously (Zola-Morgan et al., 1992; Alvarez et al., 1995). Recognition memory was assessed using two different tasks: the delayed nonmatching to sample task (the ISC, RF1, RF2, and IBO1 groups) and the visual paired-comparison task (the RF2 and IBO2 groups).

\section{MATERIALS AND METHODS}

\section{Subjects}

Behavioral findings from 27 male cynomolgus monkeys (Macaca fascicularis) are presented (see Table 2). Twenty-two monkeys sustained lesions of the hippocampal region, and five monkeys served as control animals. The animals weighed between 3.9 and $5.8 \mathrm{~kg}$ at the start of testing and belonged to six different groups. The findings from two groups have been reported previously: four monkeys with ischemic lesions of the hippocampal region [ISC group; Zola-Morgan et al. (1992)] and four monkeys with radio-frequency lesions of the hippocampal region [RF1 group; group $\mathrm{H}$ in Alvarez et al. (1995)]. The findings from three new operated groups and a control group are described in the present paper. Two groups of monkeys (IBO1 and $\mathrm{IBO} 2$ ) received bilateral ibotenate lesions of the hippocampal region (hippocampal cell fields, dentate gyrus, and subiculum). The IBO1 group $(n=5)$ was tested on the delayed nonmatching to sample task. The IBO2 group $(n=4)$ was tested on the visual paired-comparison task. The lesions in the two IBO groups were intended to damage the cell bodies of the hippocampal region but to spare white matter and adjacent medial temporal lobe structures (the amygdala and entorhinal, perirhinal, and parahippocampal cortices). Finally, five monkeys received bilateral radio-frequency lesions of the hippocampal region (RF2 group). This group was given both the delayed nonmatching task and the visual paired-comparison task. Five unoperated normal monkeys ( $\mathrm{N}$ group) were also given both tasks.

\section{Surgery}

ISC group. The procedure for making the ISC lesion has been described in detail previously (Zola-Morgan et al., 1992). Each monkey was subjected to $15 \mathrm{~min}$ of reversible ischemia, using a noninvasive technique that combined carotid occlusion and pharmacologically induced hypotension.

RF1 and RF2 groups. The procedure for making the lesions in the RF1 and RF2 groups has been described in detail previously (Alvarez-Royo et al., 1991). First, four small glass beads filled with a radio-opaque solution $\left(0.5 \mathrm{M} \mathrm{CuSO}_{4}\right)$ were anchored to the monkey's skull with dental acrylic. Magnetic resonance images (MRIs) of each animal's brain were subsequently obtained by placing the monkeys in a custom-built nonmetallic acrylic stereotaxic headholder. The radio-opaque beads served as common landmarks on the MRIs from which stereotaxic coordinates for the intended lesions could be derived. At the time of neurosurgery (within 1-5 weeks after the MRI procedure), monkeys were again placed in the headholder, and openings in the skull were made, directly overlying the hippocampal region. Then, by using a specially designed electrode connected to a radio-frequency lesion maker (Burlington, model RGF-4), seven overlapping lesions were produced along the rostrocaudal extent of the hippocampal region on each side of the brain. To avoid damaging the amygdala, we intended to spare the most anterior portion of the hippocampal region $(\sim 2-4 \mathrm{~mm})$. A similar procedure was used to make the lesions in the RF2 group.

IBO1 and IBO2 groups. The animals in the two IBO groups were prepared using imaging and surgical procedures similar to those described for the RF groups. At the time of neurosurgery, monkeys were placed in the headholder, and openings in the skull were made, directly overlying the hippocampal region. A $10 \mu$ l Hamilton syringe, filled with ibotenic acid (Biosearch Technologies, San Rafael, CA; $10 \mathrm{mg} / \mathrm{ml}$ in a 0.1 M phosphate buffer solution), was used to produce six to seven bilateral overlapping lesions along the rostrocaudal extent of the hippocampal region. At each lesion site, $0.8 \mu \mathrm{l}$ of ibotenic acid was slowly injected during $5 \mathrm{~min}$. The IBO1 group was prepared using our original acrylic stereotax and the glass bead procedure. The IBO2 group was prepared using a newer acrylic stereotax (Crist Instruments, Damascus, MD) without the glass bead procedure.

\section{Behavioral testing}

Trial-unique delayed nonmatching to sample. Testing on the trial-unique delayed nonmatching to sample task was performed in a modified version of a Wisconsin general test apparatus (Harlow and Bromer, 1938). Six to eight weeks after surgery, monkeys in the ISC, RF1, RF2, and IBO1 groups were given four to six daily sessions of pretraining to habituate them to the testing apparatus. The $\mathrm{N}$ group was pretrained in the same way. Monkeys learned to obtain food by displacing objects that covered any of the three food wells located on a stimulus tray in front of the testing chamber. All groups, except the IBO1 group (see below), were experimentally naive before behavioral testing on the delayed nonmatching to sample task.

For the nonmatching task, monkeys were first required to displace an object (the sample object) covering the central food well of a three-well stimulus tray to obtain a food reward. Then, an opaque door was lowered to block the monkeys' view of the food wells for $8 \mathrm{sec}$. After this delay interval, the monkeys saw two objects (the previously presented sample object and a novel object), each covering one of the two lateral food wells. The monkeys' task was to displace the novel object to obtain a food reward. The position of the correct object (over the left or right lateral well) varied on each trial according to a pseudorandom schedule (Gellerman, 1933). Twenty trials were presented on each day, with an intertrial interval of $20 \mathrm{sec}$. Each trial used a new pair of objects selected from a collection of more than 400 junk objects. After reaching a learning criterion of 90 correct choices in 100 trials, the monkeys were tested at successively longer delay intervals of $15 \mathrm{sec}, 1 \mathrm{~min}, 10 \mathrm{~min}$, and $40 \mathrm{~min}$. One hundred trials were administered at the $15 \mathrm{sec}$ and 1 min delay intervals ( 20 trials per day for $5 \mathrm{~d}$ ), and 50 trials were administered at the 10 and 40 min delays (five trials per day for $10 \mathrm{~d}$ ). The monkeys in the ISC group were not tested at the $40 \mathrm{~min}$ delay interval. Four to nine months later, the delayed nonmatching to sample task was administered to all groups again in exactly the same way. Between the first and second administrations of the delayed nonmatching task, all groups were given the same sequence of three other behavioral tasks, and the findings from these other tasks will be reported elsewhere.

Visual paired-comparison task. This task was given to the RF2, IBO2, and $\mathrm{N}$ groups. For the RF2 and $\mathrm{N}$ groups, the task was given after completion of the test sequence just described. The IBO2 group was given only this task. [As an exploratory procedure, the IBO1 group had been given this task preoperatively and then again immediately after surgery. However, the data were difficult to interpret because no other groups were tested in this way, and preoperative experience can influence the behavioral effects of medial temporal lobe lesions (ZolaMorgan and Squire, 1986; Murray, 1990)]. Each monkey was placed in a custom-designed Plexiglas primate chair, which allowed free movement of the arms and legs. Visual stimuli were presented on a back-projection screen located $41 \mathrm{~cm}$ in front of the monkey. The monkeys' eye movements during testing were recorded on videotape for later analysis.

During each of $5 \mathrm{~d}$ before formal testing, animals were habituated to the testing conditions. Beginning on the sixth day, 10 trials were presented each day for $4 \mathrm{~d}$ (a total of 120 different visual stimuli). Each trial had two parts. During the familiarization phase, monkeys were first presented with two identical black and white line drawings, side by side (separated by $11 \mathrm{~cm}$ ) on the screen. Monkeys were allowed to look at the pictures until they accumulated a total of $25 \mathrm{sec}$ of viewing time. Then there was a variable delay interval ( $1 \mathrm{sec}, 10 \mathrm{sec}, 1 \mathrm{~min}$, or $10 \mathrm{~min}$ ) during which the screen was blank. After the delay, during the test phase, monkeys were presented with pictures of the old stimulus and a novel stimulus, side by side. They viewed these pictures until they accumulated a total of $5 \mathrm{sec}$ of viewing time. Then, after a $1 \mathrm{sec}$ interval, monkeys were presented with pictures of the same old stimulus and a different novel stimulus for an additional $5 \mathrm{sec}$ of total viewing time. The position of the old stimulus was left, then right for half of the trials and right, then left for the other half. Each of the 4 testing days used a different delay interval between the familiarization phase and the test phase $(1 \mathrm{sec}$ to 10 $\mathrm{min}$ ), and the delays were presented in pseudorandomized order across days. New sets of stimuli were used for each trial.

For each monkey, the percentage of time spent viewing the novel stimulus was calculated. The score was determined by a rater, blind to the locations of the old and novel stimuli, who counted the number of frames in which the subject looked at the stimulus on the left side or the stimulus 
on the right side. A second blind rater also scored each videotape session (within-session inter-rater reliability was $r=0.95$; range, 0.91-0.99).

\section{Neurohistological methods}

The histological procedures for the IBO1, IBO2, and RF2 groups were similar to the procedures described previously for the ISC and RF1 groups (Zola-Morgan et al., 1992; Alvarez et al., 1995) and are here described briefly. Monkeys were administered an overdose of Nembutal and perf used transcardially with $200 \mathrm{ml}$ of a buffered $0.9 \% \mathrm{NaCl}$ solution followed by 21 of $10 \%$ formaldehyde solution (in $0.1 \mathrm{M}$ phosphate buffer) at a rate of $100 \mathrm{ml} / \mathrm{min}$. Brains were then blocked in situ in the coronal plane, removed from the skull, cryoprotected first in a $10 \%$ glycerol $/ 10 \%$ formaldehyde solution (in $0.1 \mathrm{M}$ phosphate buffer) and then in a $20 \%$ glycerol $/ 10 \%$ formaldehyde solution, and subsequently quick-frozen in isopentane at $-78^{\circ} \mathrm{C}$. Coronal sections $(50 \mu \mathrm{m})$ were cut with a freezing microtome beginning just anterior to the hippocampus and continuing caudally through the length of the hippocampal region. Every fifth section was mounted and stained with thionin to assess the extent of the lesions.

Determination of the amount of damage to the hippocampal region. For each monkey in the ISC group, thionin-stained sections were examined at $0.96 \mathrm{~mm}$ intervals along the rostrocaudal extent of the hippocampal region. Camera lucida drawings of the perimeter of the CA1 field were then made from each slide at a $30 \times$ magnification and traced using a digitizing tablet to compute an areal measurement for each section. For each brain, the measurements for each level were added together, and the sum was multiplied by the interslice interval $(0.96 \mathrm{~mm})$ to obtain an estimate of the spared CA1 volume. The overall measurements of CA1 volume in the ISC group were compared with measurements of CA1 volume obtained from four weight-matched, unoperated control monkeys.

For each monkey in the RF1, RF2, IBO1, and IBO2 groups, thioninstained sections were examined at $0.5 \mathrm{~mm}$ intervals along the rostrocaudal extent of the hippocampal region. Each section was scanned into a Power Macintosh G3 computer using a ScanMaker 4 scanner. The structures comprising the hippocampal region (the dentate gyrus, the cell fields of the hippocampus proper, and the subiculum) were classified on the basis of cytoarchitectonics using a light microscope (Leica WILD $3 \mathrm{Z}$ ), and the boundaries for the hippocampal region were marked on the computerized images of each section. Using the NIH Image program and Canvas, bilateral measures of the cross-sectional area of the hippocampal region were obtained from each section. For each brain, the cross-sectional area for each section was multiplied by the interslice interval and added together to obtain a measure of the volume of the spared hippocampal region. Then, the overall measure of spared hippocampal region volume for each monkey with IBO or RF lesions was compared with the average measures of hippocampal region volume from three weight-matched, unoperated control monkeys to obtain a measure of percentage of damage. The percentages of damage to the regions that included the CA1/subiculum, the CA3/dentate gyrus, the anterior half of the hippocampal region, and the posterior half of the hippocampal region were also determined, using the procedures just described.

Determination of the amount of damage to the parahippocampal cortex and the tail of the caudate nucleus. For each monkey in the IBO1 group, brain sections were examined at $1 \mathrm{~mm}$ intervals along the rostrocaudal extent of the temporal lobe (range, 16-19 sections), and the same procedures used to determine the extent of damage to the hippocampal region were used to determine the percentage of damage to the parahippocampal cortex. Damage to the parahippocampal cortex in monkeys RF1-3 and IBO2-1 was also measured in this way. The parahippocampal cortex was entirely spared in all other monkeys in the study.

The same procedures described for determining the extent of damage to the hippocampal region were also used for determining the extent of damage to the tail of the caudate nucleus. The overall estimates of spared caudate nucleus volume for the monkeys with IBO2 and RF2 lesions were compared with estimates of the caudate nucleus volume obtained from a group of three weight-matched, unoperated control monkeys.

\section{RESULTS}

\section{Neurohistological findings (Table 1, Figs. 1-6)}

\section{The ISC and RF1 groups}

Neurohistological findings from the monkeys in the ISC and RF1 groups have been published previously [ISC: Zola-Morgan et al. (1992; RF1: group H in Alvarez et al. (1995)]. Briefly, the four monkeys with ISC lesions sustained significant loss of pyramidal cells in the CA1 and CA2 fields of the hippocampus, as well as loss of somatostatin-immunoreactive cells in the hilar region of the dentate gyrus. Cell loss occurred bilaterally throughout the rostrocaudal extent of the hippocampus but was greater in the caudal portion. The damage within the CA1 pyramidal cell field averaged $24 \%$ of total CA1 volume in three of the monkeys and $73 \%$ in the monkey with the largest lesion (ISC2) (Table 1). Animal ISC2 also sustained some subicular damage. Except for patchy loss of cerebellar Purkinje cells, significant damage was not detected in areas outside the hippocampus, including the adjacent entorhinal, perirhinal, and parahippocampal cortices. For the four monkeys in the RF1 group, the mean percentage of damage to the hippocampal region was $62 \%$ (range, 47-76\%). [To maintain consistency in the percentage of damage measures across operated groups, the brains of the RF1 group were remeasured in the present study using the three control brains that were used to obtain the percentage of damage measures for the IBO1, IBO2, and RF2 groups. Differences between the values reported for brain areas in Alvarez et al. (1995) and the present study averaged $4.5 \%$ ]. The perirhinal cortex was spared in all of the monkeys. There was damage to the entorhinal cortex in one monkey (RF1-3, $\sim 10 \%$ overall damage), and this monkey also sustained slight to moderate damage to the parahippocampal cortex $(38 \%)$. Entorhinal cortex was spared in the other three monkeys. Slight to moderate asymmetrical damage to white matter subjacent to the hippocampal region occurred in three animals (RF1-1, RF1-2, and RF1-3), and unilateral damage to the tail of the caudate nucleus occurred in two animals (RF1-1 and RF1-2).

\section{IBO1 group}

Overall, the five monkeys in the IBO1 group sustained substantial bilateral damage to the hippocampal region (i.e., the cell fields of the hippocampus proper, the dentate gyrus, and the subiculum). The mean percentage of damage for all five animals was $44 \%$ (range, 34-53\%) (Table 1). The most anterior portion of the hippocampal region was intentionally spared to prevent inadvertent damage to the amygdala, and the amygdala was entirely spared in all five animals. The entorhinal and perirhinal cortices sustained slight unilateral damage in one animal (IBO1-5) but were spared in the other four animals. The parahippocampal cortex was completely spared in monkey IBO1-2. There was slight to moderate unilateral damage to the posterior parahippocampal cortex in two animals (amounting to $\sim 10 \%$ in IBO1-1 on the left side and $23 \%$ in IBO1-5 on the right side) and slight to moderate bilateral damage to the parahippocampal cortex in two animals (amounting to $\sim 7 \%$ in monkey IBO1-3 and $46 \%$ in monkey IBO1-4). There was no damage to the tail of the caudate nucleus, the stria terminalis, or the lateral geniculate nucleus in any of the animals. Figure 1 illustrates the extent of damage in each of the IBO1 monkeys, plotted on representative coronal sections adapted from Szabo and Cowan (1984), and Figure 2 shows photomicrographs from monkey IBO1-2.

\section{IBO2 group}

The mean percentage of hippocampal damage for all four animals was $33 \%$ (range, 13-60\%) (Table 1). As in the IBO1 group, the lesions were intended to spare the most anterior portion of the hippocampal region to prevent inadvertent damage to the amygdala. However, in two of the four animals (IBO2-2 and IBO2-3), the damage began more anterior than intended, and these two 


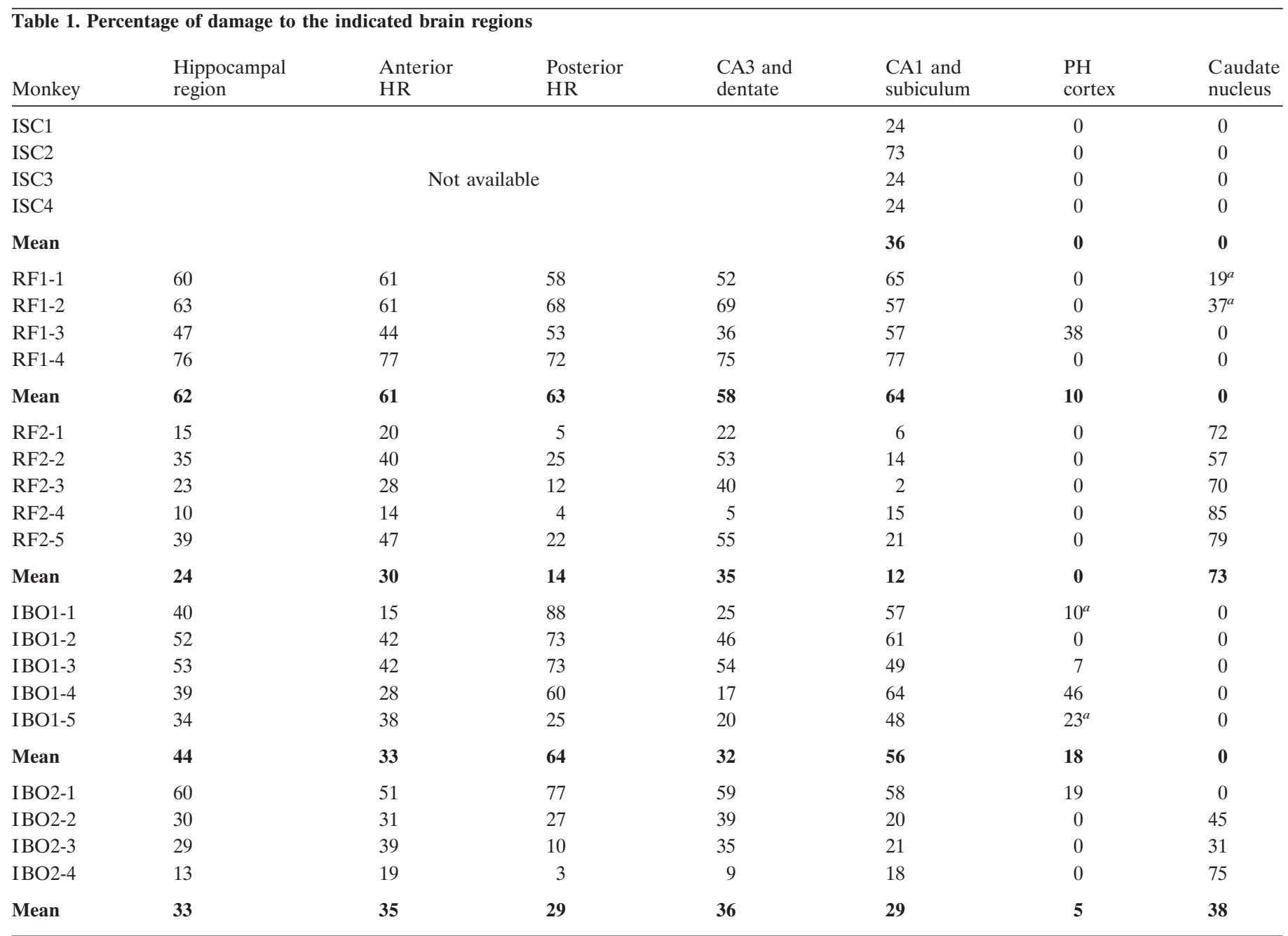

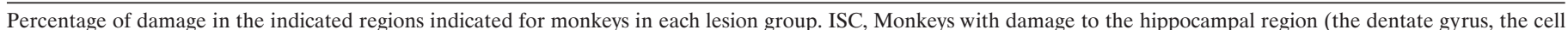

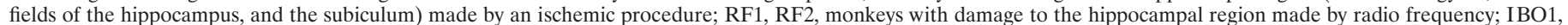

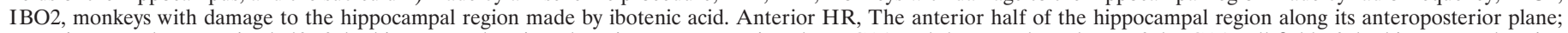

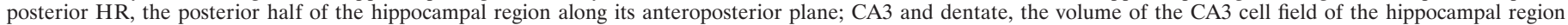

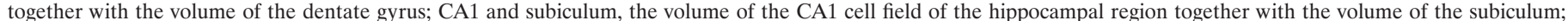

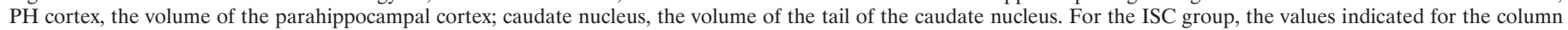
headed "CA1 and subiculum" are the percentage of total CA1 volume.

${ }^{a}$ Indicates unilateral damage.

animals had slight unilateral (IBO2-3 on the left side) or slight bilateral (IBO2-2) damage to the most posterior aspect of the amygdala. In the other two animals (IBO2-1 and IBO2-4), the amygdala was completely spared. The entorhinal cortex and perirhinal cortex were spared in all four animals. Monkey IBO2-1 sustained bilateral damage to the posterior parahippocampal cortex (amounting to $\sim 19 \%$ ). In three animals, there was also bilateral damage to the tail of the caudate nucleus (amounting to $\sim 45 \%$ in IBO2-2, 31\% in IBO2-3, and $75 \%$ in IBO2-4). There was slight to moderate damage to the lateral geniculate nucleus in two of the monkeys ( $\sim 13 \%$ in IBO2-3 and $\sim 11 \%$ in IBO2-4). Monkey IBO2-1 also sustained slight unilateral damage to the medial portion of area TEO subjacent to the most posterior portion of the right hippocampal region. Figure 3 illustrates the extent of damage in each of the IBO2 monkeys, plotted on representative coronal sections adapted from Szabo and Cowan (1984), and Figure 4 shows photomicrographs from monkey IBO2-1.

\section{RF2 group}

The mean percentage of damage for all five animals was $24 \%$ (range, 10-39\%) (Table 1). In all five animals, the damage to the hippocampal region was limited mainly to field CA3 and to the dentate gyrus. As in the IBO groups, the lesions were intended to spare the most anterior portion of the hippocampal region to prevent inadvertent damage to the amygdala. However, in three of the animals (RF2-1, RF2-2, and RF2-4), the damage began more anterior than intended. Two of these animals (RF2-1 and RF2-2) had slight bilateral damage to the posterior portion of the amygdala, and one animal (RF2-4) had moderate bilateral damage to the posterior amygdala. The entorhinal, perirhinal, and parahippocampal cortices were spared in all five animals. There was bilateral damage to the tail of the caudate nucleus in all five animals (mean percentage of damage $=73 \%$ ), and all five animals sustained slight to moderate bilateral damage to the lateral aspect of the lateral geniculate nucleus (range, 19-45\%). There was also slight to moderate bilateral damage to the anterior 
IB01-1

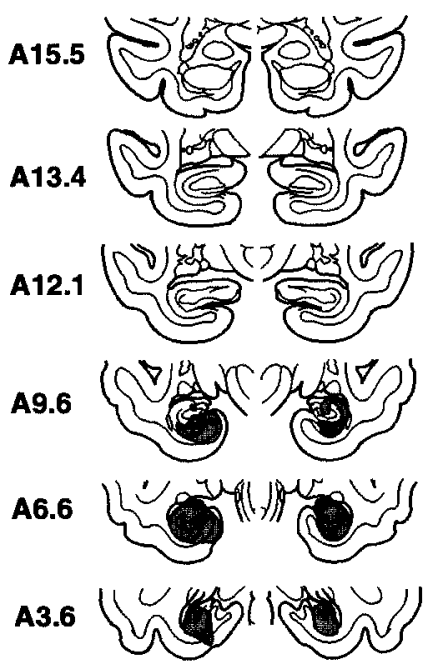

IB01-2
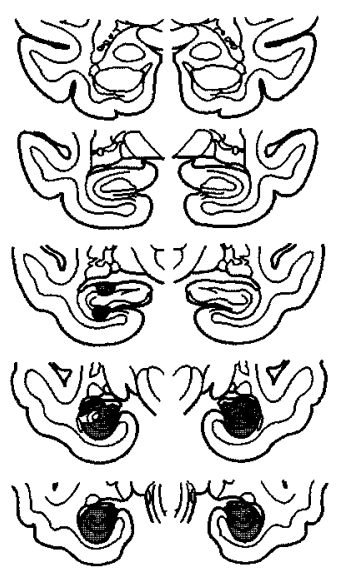

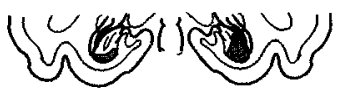

IB01-3
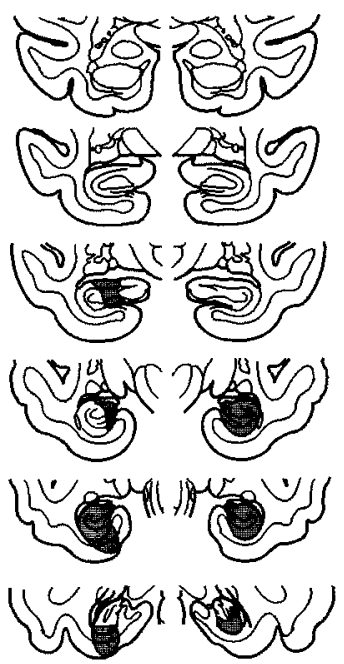

IB01-4
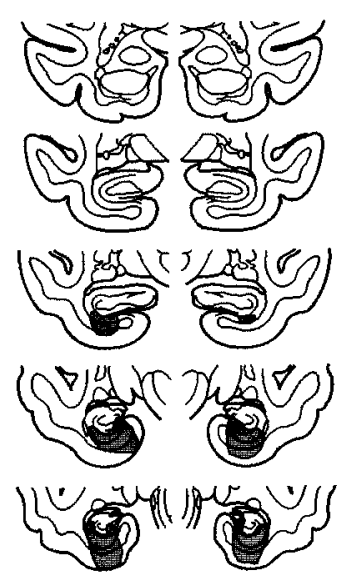

urats
IB01-5
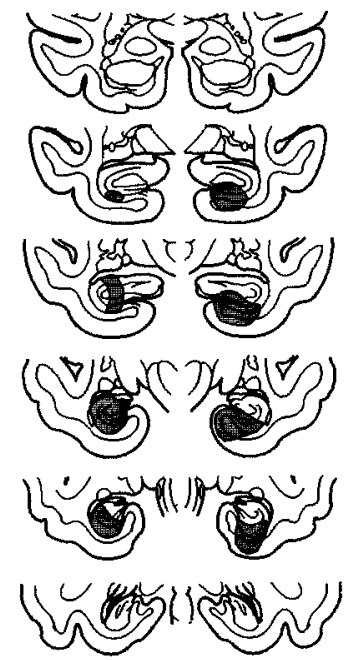

Figure 1. Group IBO1. Line drawings of representative coronal sections through the temporal lobe of $M$. fascicularis adapted from the atlas of Szabo and Cowan (1984). The sections are arranged from rostral (A15.5) to caudal $(A 3.6)$. The designations $A 15.5, A 13.4$, and so on specify distances anterior to the intra-aural line in millimeters. The extent of the lesions of the hippocampal region in each of the five monkeys in the IBO1 group is plotted on the coronal sections. In each case, the area of the lesion is indicated in gray.

portion of the stria terminalis in all five monkeys. Figure 5 illustrates the extent of damage in each of the RF2 monkeys, plotted on representative coronal sections adapted from Szabo and Cowan (1984), and Figure 6 shows photomicrographs from monkey RF2-5.

\section{Behavioral findings}

Table 2 shows the performance scores for the monkeys in the N, ISC, RF1, RF2, IBO1, and IBO2 groups on the two recognition memory tasks: trial-unique delayed nonmatching to sample and visual paired-comparison. All analyses are based on paired, unpaired, or one-sample $t$ tests.

\section{The delayed nonmatching to sample task}

This task was administered to the N, ISC, RF1, RF2, and IBO1 groups on two different occasions, separated by 4-9 months (see Materials and Methods). On the first administration of the task, the learning scores of the $\mathrm{N}$ group (mean trials to criterion level of performance: 118 trials) were similar to the learning scores of the lesion groups $(\mathrm{ISC}=50$ trials, RF1 $=290$ trials, RF2 $=352$ trials, $\mathrm{IBO} 1=19$ trials; all $t$ values $<1.98$, all $p$ values $>0.05)$. On the second administration of the task, the $\mathrm{N}$ group (20 trials) again performed like the lesion groups (ISC $=85$ trials, $\mathrm{RF} 1=25$ trials, $\mathrm{RF} 2=25$ trials, $\mathrm{IBO} 1=0$ trials; all $p$ values $>0.09$ ).

Figure 7 shows performance of all groups on the delay portion of the delayed nonmatching to sample task averaged across the two test administrations. In each panel, asterisks signify impaired performance $(p<0.05)$ of the lesion group relative to the five normal control monkeys $(\mathrm{N})$. All four lesion groups performed rather similarly overall. For example, performance was nearly identical at the 10 min delay interval (ISC $=64 \%$ correct, $\mathrm{RF} 1=$ $69 \%$ correct, $\mathrm{RF} 2=66 \%$ correct, and $\mathrm{IBO} 1=69 \%$ correct; $p>$ $0.10)$ and also at the 40 min delay interval (RF1 $=62 \%$ correct, $\mathrm{RF} 2=61 \%$ correct, and $\mathrm{IBO} 1=63 \%$ correct $)$.

\section{The visual paired-comparison task}

Figure 8 presents data for the RF2 group (left) and the IBO2 group (right). The $\mathrm{N}$ group consists of the same five monkeys that were tested in the delayed nonmatching to sample task. In each panel, asterisks signify impaired performance $(p<0.05)$ of the lesion group relative to the $\mathrm{N}$ group. At the $1 \mathrm{sec}$ delay, the RF2 and IBO2 groups viewed the novel picture as much as the $\mathrm{N}$ group ( $\mathrm{RF} 2=65 \%$ of time viewing the novel picture; IBO2 group $=64 \%, n=65 \%$ ). This observation indicates that the lesions did not interfere with perception or the ability to respond to novelty per se. When the delay interval was increased beyond $1 \mathrm{sec}$, performance of both the RF2 group and the IBO2 group differed from the $\mathrm{N}$ group at every delay (except the RF2 group at $10 \mathrm{sec}, p=0.119$, and the IBO2 group at $1 \mathrm{~min}, p=0.057)$. The RF2 group performed overall above chance [57\% across the three delay intervals, $p<0.05 ; 10 \mathrm{sec}(p=0.06), 1 \mathrm{~min}(p<0.001)$, and $10 \mathrm{~min}(p<0.05)]$. The IBO2 group performed similarly to the RF2 group (55 vs 57\%, respectively, across the three delays), although the IBO2 group's performance at each of the three delays, as well as their overall performance, was not significantly above chance $(p>0.05)$. There was no evidence of forgetting in the $\mathrm{N}$ group across the delays tested (1 sec delay: $65 \%$ vs $10 \mathrm{~min}$ delay: $62 \% ; p>.10$ )

\section{Relationship between extent of damage and behavioral scores (Tables 1, 2)}

Four monkeys in the IBO1 group as well as monkeys RF1-3 and IBO2-1 sustained some inadvertent damage to the parahippocampal cortex in addition to the intended damage to the hippocampal region. Two of these monkeys also sustained slight damage to the entorhinal cortex (RF1-3 and IBO1-5), and one of these sustained slight damage to perirhinal cortex (IBO1-5). Moreover, three monkeys in the IBO2 group and all five monkeys in the RF2 group sustained inadvertent damage to the caudate nucleus. To evaluate the possible contribution of this damage to behavioral impairment, we performed additional analyses.

With regard to the parahippocampal cortex, entorhinal cortex, and perirhinal cortex, we compared performance on the delayed nonmatching task for the five monkeys that sustained any damage to these cortices (four monkeys in the IBO1 group and monkey 

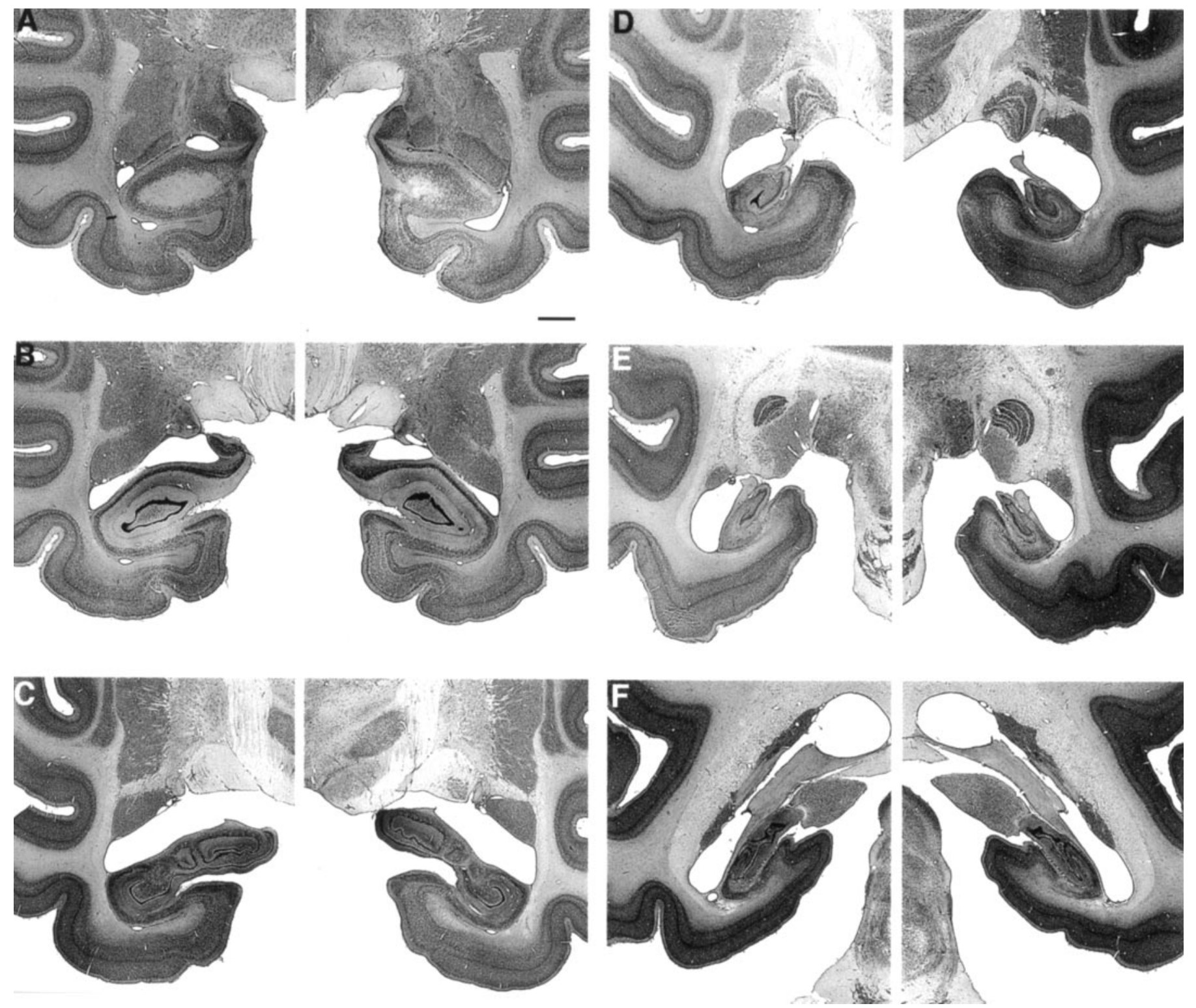

Figure 2. Photomicrographs of thionin-stained sections through the left and right temporal lobe of monkey IBO1-2, whose lesion approximated the intended lesion. The sections are arranged from rostral $(A)$ to caudal $(F)$ and correspond to the line drawings in Figure 1 . Scale bar (shown in $A$ for $A-F$ ): $2 \mathrm{~mm}$.

RF1-3) with the performance of the remaining 13 monkeys with lesions of the hippocampal region but without damage to adjacent cortex (ISC, $n=4$; RF1, $n=3$; RF2, $n=5$; IBO1, $n=1$ ). Performance across the $15 \mathrm{sec}, 1 \mathrm{~min}$, and $10 \mathrm{~min}$ delays was quite similar in the two groups (five monkeys that sustained adjacent cortical damage: $84 \%$ correct; remaining 13 monkeys: $81 \%$ correct; $p>0.10)$. It is also notable that all four monkeys in the IBO1 group that sustained damage to adjacent cortex obtained higher average scores across the $15 \mathrm{sec}, 1 \mathrm{~min}$, and $10 \mathrm{~min}$ delays than the IBO1 monkey with sparing of adjacent cortex (Tables 1, 2). Additionally, compared with the five control monkeys, the 13 monkeys with hippocampal damage but no damage to adjacent cortex were significantly impaired at the $10 \mathrm{~min}$ delay of the delayed nonmatching task (the 13 monkeys scored $65 \%$ correct; the five control monkeys scored $79 \%$ correct; $p<0.001$ ), and they were also impaired across the $15 \mathrm{sec}, 1 \mathrm{~min}$, and $10 \mathrm{~min}$ delays (the 13 monkeys scored $81 \%$ correct; the five control monkeys scored $89 \%$ correct; $p<0.001)$. Thus, insofar as we could determine, there was no evidence that inadvertent cortical damage adjacent to the hippocampus contributed in a systematic way to the impaired performance.

With regard to the caudate nucleus, we performed several analyses. For the delayed nonmatching to sample task, we compared the scores of the five monkeys in the RF2 group and the two monkeys in the RF1 group that sustained caudate damage with the scores of the 11 remaining monkeys that had no caudate damage (ISC, $n=4$; RF1, $n=2$; IBO1, $n=5$ ). The scores for these two groups of monkeys, combined across the $15 \mathrm{sec}, 1 \mathrm{~min}$, and 10 min delays, were identical (seven monkeys with caudate nucleus damage: $82 \%$ correct; remaining 11 monkeys: $82 \%$ correct). We also compared the scores of the seven RF monkeys that sustained caudate damage with the scores of the two RF monkeys who sustained the same type of lesion but without caudate damage (RF1-3 and RF1-4). The scores for these two groups were 
IBO2-1

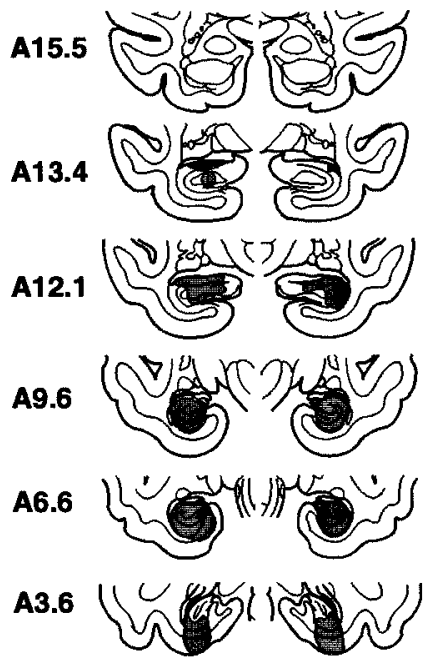

IBO2-2

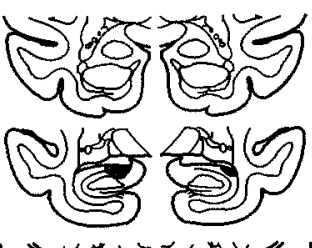

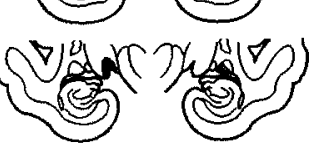

IBO2-3
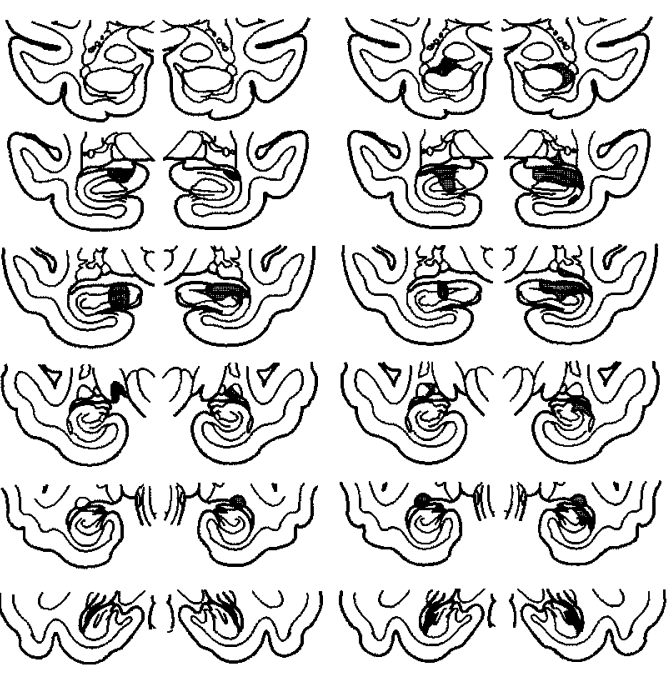

IBO2-4
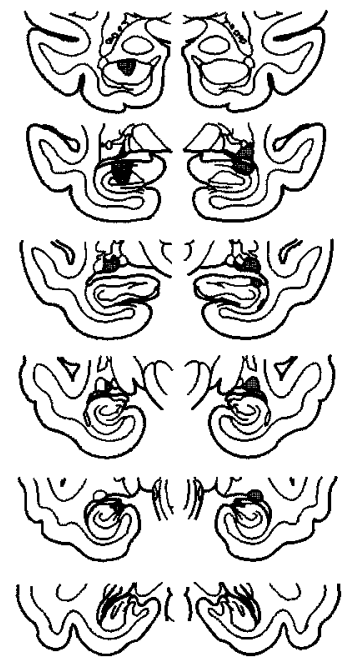

Figure 3. Group IBO2. The extent of the lesions of the hippocampal region in each of the four monkeys in the IBO2 group is indicated in gray on the coronal sections. Sections are arranged as in Figure 1.

also nearly identical (average difference across $15 \mathrm{sec}, 1 \mathrm{~min}, 10$ min, and 40 min delays: $2.5 \%$ ). Finally, the 11 monkeys without caudate damage were impaired on the nonmatching to sample task when compared with the five normal monkeys in Figure 7 [15 sec: $\mathrm{N}$ group $=94 \%$ correct, 11 monkeys with caudate damage $=90 \%$ correct, $p<0.04 ; 1 \mathrm{~min}: n=92 \%$ correct, 11 monkeys $=88 \%$ correct, $p=0.071 ; 10 \min : n=79 \%$ correct, 11 monkeys $=67 \%$ correct, $p<0.01 ; 40 \mathrm{~min}: n=75 \%$ correct, 7 monkeys (the four ISC monkeys were not tested at this delay) $=62 \%$ correct, $p<0.01]$.

For the visual paired-comparison task, we could only compare the scores of the single monkey who had no damage to the caudate nucleus (IBO2-1) with the scores of the other monkeys that sustained caudate nucleus damage. This monkey performed within $1 \%$ of the average score of the other monkeys in the IBO2 group and within 3\% of the average score of all eight monkeys that sustained caudate damage (IBO2, $n=3$; RF2, $n=5$ ). Thus, insofar as we could determine, there was no suggestion that inadvertent damage to the caudate nucleus contributed to the impaired performance of the IBO2 or the RF2 groups.

Finally, we considered separately the performance on the delayed nonmatching task of the six monkeys who sustained hippocampal damage but no other extraneous damage, no matter how minimal (the four ISC monkeys and monkeys RF1-4 and IBO1-2). Compared with the five control monkeys, these six monkeys with hippocampal lesions were significantly impaired at the 10 min delay of the delayed nonmatching task (the six monkeys that sustained only hippocampal damage scored $64 \%$ correct; the five control monkeys scored $79 \%$ correct, $p<0.01$ ), and they were also impaired across the $15 \mathrm{sec}, 1 \mathrm{~min}$, and $10 \mathrm{~min}$ delays (the six monkeys that sustained only hippocampal damage scored $79 \%$ correct; the five control monkeys scored $89 \%$ correct; $p<0.01)$.

In the present study, the locus and extent of damage to the hippocampal region was variable both within and between groups (Table 1). This circumstance, together with the unusually large number of operated monkeys $(n=22)$, provided an opportunity to assess the relationship between the locus and extent of damage to the hippocampal region and recognition memory performance. We first considered the relationship between extent of damage to the full anteroposterior extent of the hippocampal region (combined damage to the dentate, CA1, CA3, and subiculum) and recognition memory performance on both tasks (a $z$-score for each monkey was derived from the percentage of correct scores of the delayed nonmatching to sample task and the visual pairedcomparisons task at the 10 min delay interval; for monkeys that were tested on both tasks, the mean of the two $z$-scores was used). There was no correlation between extent of damage to the hippocampal region and recognition memory performance $(r=0.05$, $p>0.10)$.

Recent evidence from rats suggests that the hippocampal region can be functionally differentiated along its dorsoventral (septotemporal) axis. Specifically, rats were more impaired on spatial learning in a Morris water maze when dorsal hippocampal tissue was damaged ( $20 \%$ or more) than when equally large lesions were made in ventral hippocampus (E. Moser et al., 1993; M. B. Moser et al., 1995; for review, see Moser and Moser, 1998).

In primates, the anterior hippocampus corresponds to the rodent ventral hippocampus, and the posterior hippocampus corresponds to the rodent dorsal hippocampus. Using the strategy developed in the work with rodents, we divided the monkey hippocampal region into an anterior and a posterior segment for each animal by selecting a point approximately midway along the anterior-posterior axis. The finding was that the extent of damage in neither the anterior hippocampal region $(r=0.09)$ nor the posterior hippocampal region $(r=0.005)$ correlated with recognition memory performance (using the $z$-score measure described above; $p>0.10)$.

We also divided the hippocampal region into a dorsal component (which included the dentate gyrus and the CA3 field) and a ventral component (which included the CA1 field and the subiculum). Again, the extent of damage did not correlate with recognition memory performance $(r$ values $<0.33$, p values $>0.10)$. Thus, although damage to the hippocampal region produced impaired recognition memory, a significant correlation was not 

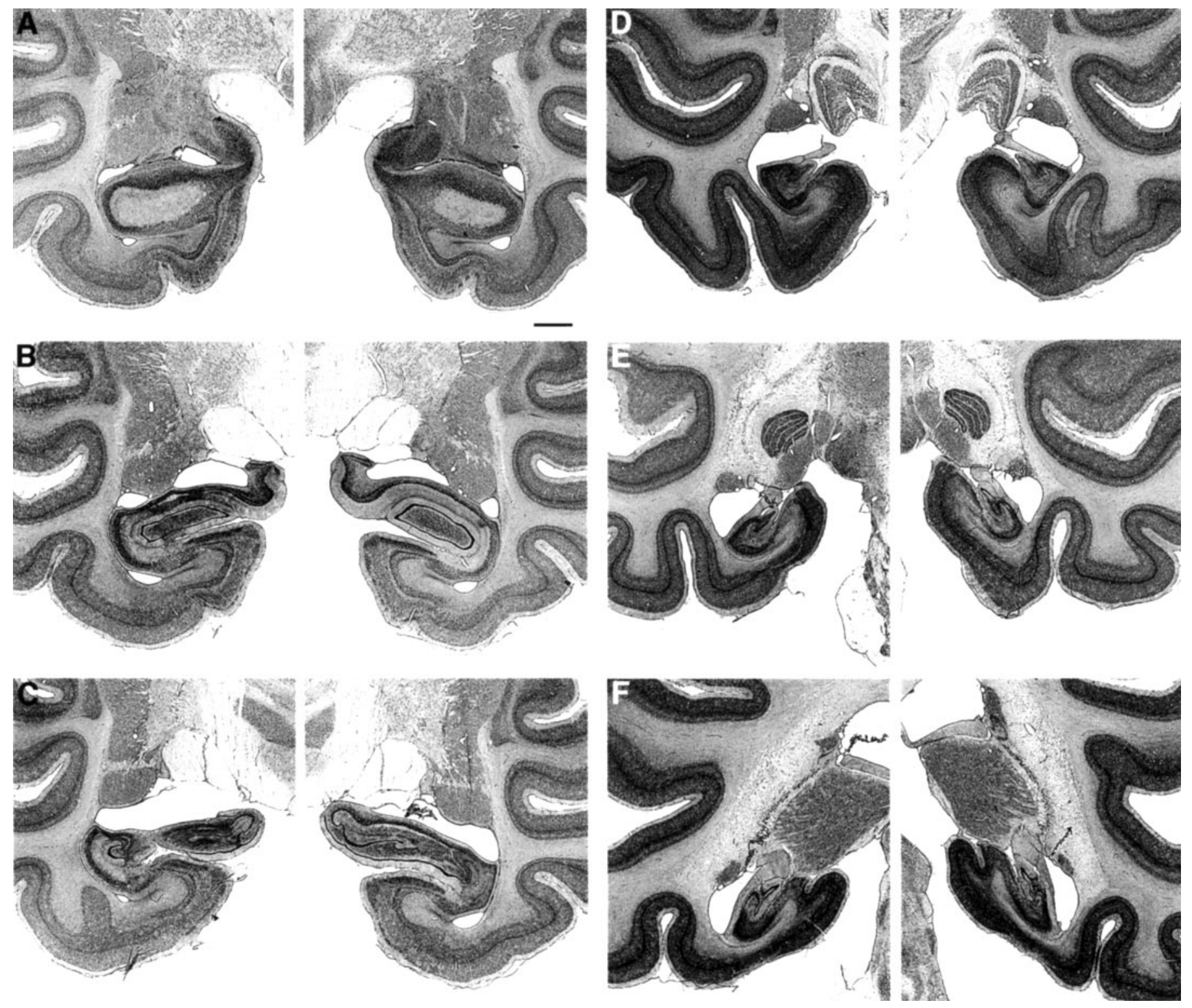

Figure 4. Photomicrographs of thionin-stained sections through the left and right temporal lobe of monkey IBO2-1, whose lesion approximated the intended lesion. The sections are arranged from rostral $(A)$ to caudal $(F)$ and correspond to the line drawings in Figure 3. Scale bar (shown in $A$ for $A-F): 2 \mathrm{~mm}$.

found between the extent of damage to the hippocampal region and recognition memory performance.

\section{DISCUSSION}

The results show that the hippocampal region is essential for normal recognition memory performance. Damage to the hippocampal region by each of three techniques impaired memory to the same extent. Thus, on the delayed nonmatching to sample task, monkeys in the ISC, RF1, RF2, and IBO-1 groups exhibited a similar level of impairment (Fig. 7). Moreover, on the visual paired-comparison task, monkeys in the RF2 and IBO2 groups exhibited a similar level of impairment (Fig. 8). The fact that monkeys with ISC or RF lesions were no more impaired on any of the performance measures than were the monkeys with IBO lesions suggests that it is unlikely that undetected damage outside the hippocampus (in the ISC group) or damage to adjacent fibers (in the RF groups) contributed substantially to impaired performance. Although some monkeys sustained unintended damage to the caudate nucleus, recognition memory performance was significantly impaired in the 11 monkeys who had no caudate damage. Moreover, in a preliminary report, monkeys with caudate nucleus lesions large enough to impair concurrent discrimination learning performed normally on the nonmatching to sample task (Wang et al., 1990).

An additional issue needs to be considered in the case of the delayed nonmatching to sample task. For practical reasons related to testing many animals at the same time, monkeys were removed from the testing apparatus and returned to their home cage during the long delays (10 and $40 \mathrm{~min}$ ) but not during the shorter delays (15 sec and $1 \mathrm{~min}$ ). In the two earliest studies (Zola- 
RF2-1

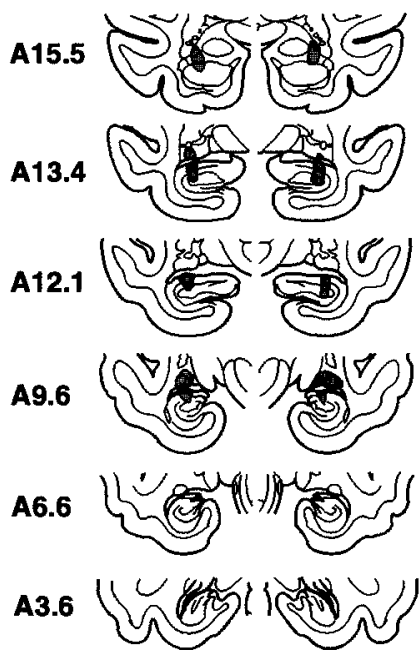

RF2-2
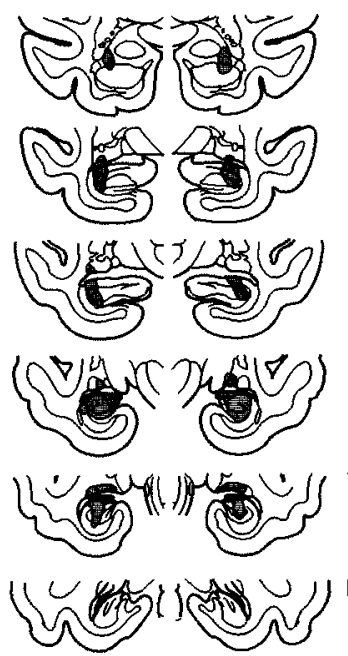

RF2-3
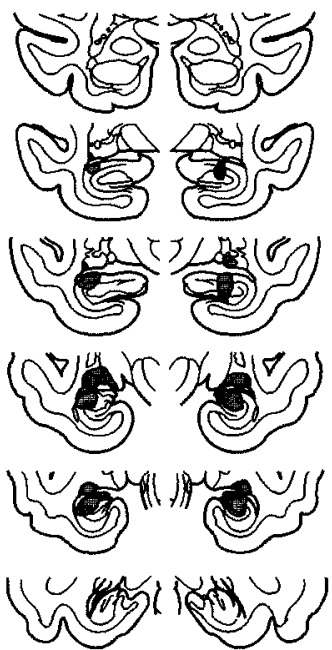

RF2-4
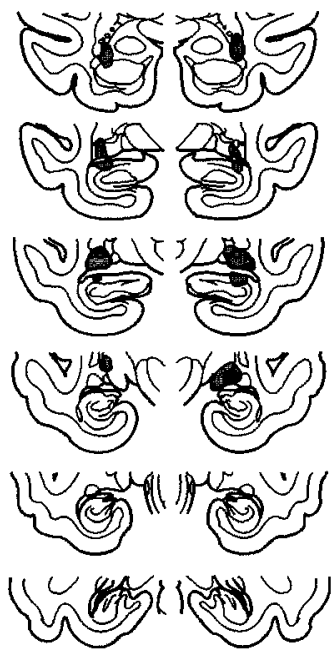

RF2-5
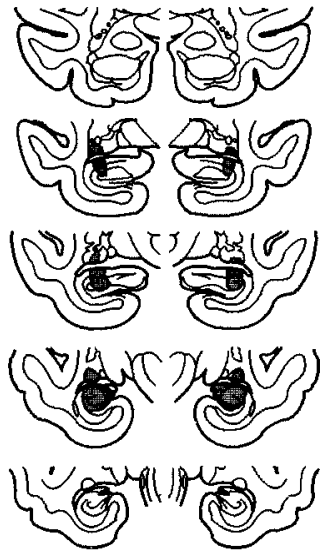

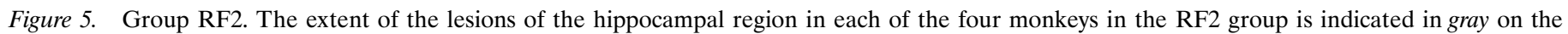
coronal sections. Sections arranged as in Figure 1.

Morgan et al., 1992; Alvarez et al., 1995), impaired performance was not usually observed at the shorter delays (Fig. 7, top two panels). This finding led to the suggestion that the deficit might reflect a deficit in spatial memory attributable to the fact that the monkeys must reorient themselves to the testing apparatus each time they are returned to it (Nadel, 1995; Murray and Mishkin, 1998).

Three observations argue against this idea. First, for the ISC, RF2, and IBO-1 monkeys, impaired performance was observed at short delay intervals ( $\leq 1 \mathrm{~min}$ ) even when monkeys remained in the test apparatus (Fig. 7). Second, in the visual pairedcomparison task, the monkeys were never removed from the test apparatus but were significantly impaired at all delays longer than $1 \mathrm{sec}$ (Fig. 8). Third, using three of the five $\mathrm{N}$ monkeys and four of the RF2 monkeys, we have compared directly the effect on delayed nonmatching to sample performance of having the animals remain in the test apparatus during the 10 min delay $(n=$ $93 \%$ correct, $\mathrm{RF} 2=73 \%$ correct) or removing animals from the test apparatus during the 10 min delay $(n=85 \%$ correct, RF2 $=$ $63 \%$ correct). Removing monkeys from the test apparatus had a similar effect on both the normal and operated monkeys (group $\times$ condition interaction, $p>0.10$ ) (Teng et al., 1998).

The effects of hippocampal lesions on recognition memory are consistent and clear. Figure 9 combines data from all of the monkeys with hippocampal damage $(\mathrm{H})$ in the current study (Figs. 7,8) together with all the data from normal monkeys who have been tested in our laboratory on either the delayed nonmatching to sample task $(n=10)$ or the visual paired-comparison task $(n=13)$. In our previous report of impaired nonmatching to sample performance after hippocampal damage (Alvarez et al., 1995), impaired performance was detected only at the long delays (10 and $40 \mathrm{~min}$ ). In the present study, it was possible to consider together the data from a much larger number of monkeys than can be done in the typical study. The finding with this large data set was that performance on the delayed nonmatching to sample task was impaired at all delays longer than $8 \mathrm{sec}$ (although to a small degree at the shorter delays). The impairment on the visual paired-comparison task was qualitatively similar to the impairment on the delayed nonmatching to sample task but was robust even at a delay of $10 \mathrm{sec}$. The findings for the two tasks are consistent with the suggestion that the visual paired-comparison task is more sensitive to hippocampal damage than nonmatching to sample (McKee and Squire, 1993).

The findings in monkeys are fully consistent with the findings from humans, where damage limited to the hippocampus is associated with moderately severe amnesia and impaired recognition memory (Zola-Morgan et al., 1986; Squire et al., 1988; RempelClower et al., 1996). When rehearsal is largely prevented by using nonverbal stimuli (e.g., flower-like patterns), impaired recognition memory in amnesic patients is apparent as early as $6-10 \mathrm{sec}$ after learning (Buffalo et al., 1998).

An exception to this pattern of findings comes from a report of three individuals who sustained hippocampal damage early in life yet performed well on standard recognition memory tests despite poor recall and otherwise poor everyday memory functioning (Vargha-Khadem et al., 1997, 1998). Yet patients with damage limited to the hippocampal formation, who became amnesic as adults, performed poorly on the same recognition memory test that one of the early-onset cases performed well (Manns and Squire, 1999). We suggest that good recognition performance after hippocampal damage is limited to lesions that occur early in life and reflects some compensatory behavioral strategy acquired during early life (Manns and Squire, 1999).

Of the five studies that report the effects on recognition memory in monkeys with lesions of the hippocampal region, and where postmortem neurohistological analyses of the lesions were provided, four have found impaired recognition memory (ZolaMorgan et al., 1992; Alvarez et al., 1995; Beason-Held et al., 1999) (and the present study). The fifth study found no impairment on the delayed nonmatching to sample task (Murray and Mishkin, 1998). This study differed from the others in potentially important ways. First, unlike the ISC, RF, and IBO monkeys in the other four studies, the IBO monkeys tested by Murray and Mishkin (1998) received preoperative training on the nonmatching task. During preoperative training, the nonmatching rule was first trained during several hundred trials using a delay interval of $8-10 \mathrm{sec}$. Training on the rule provides the monkey with extended practice at holding novel objects in memory across short delays, 

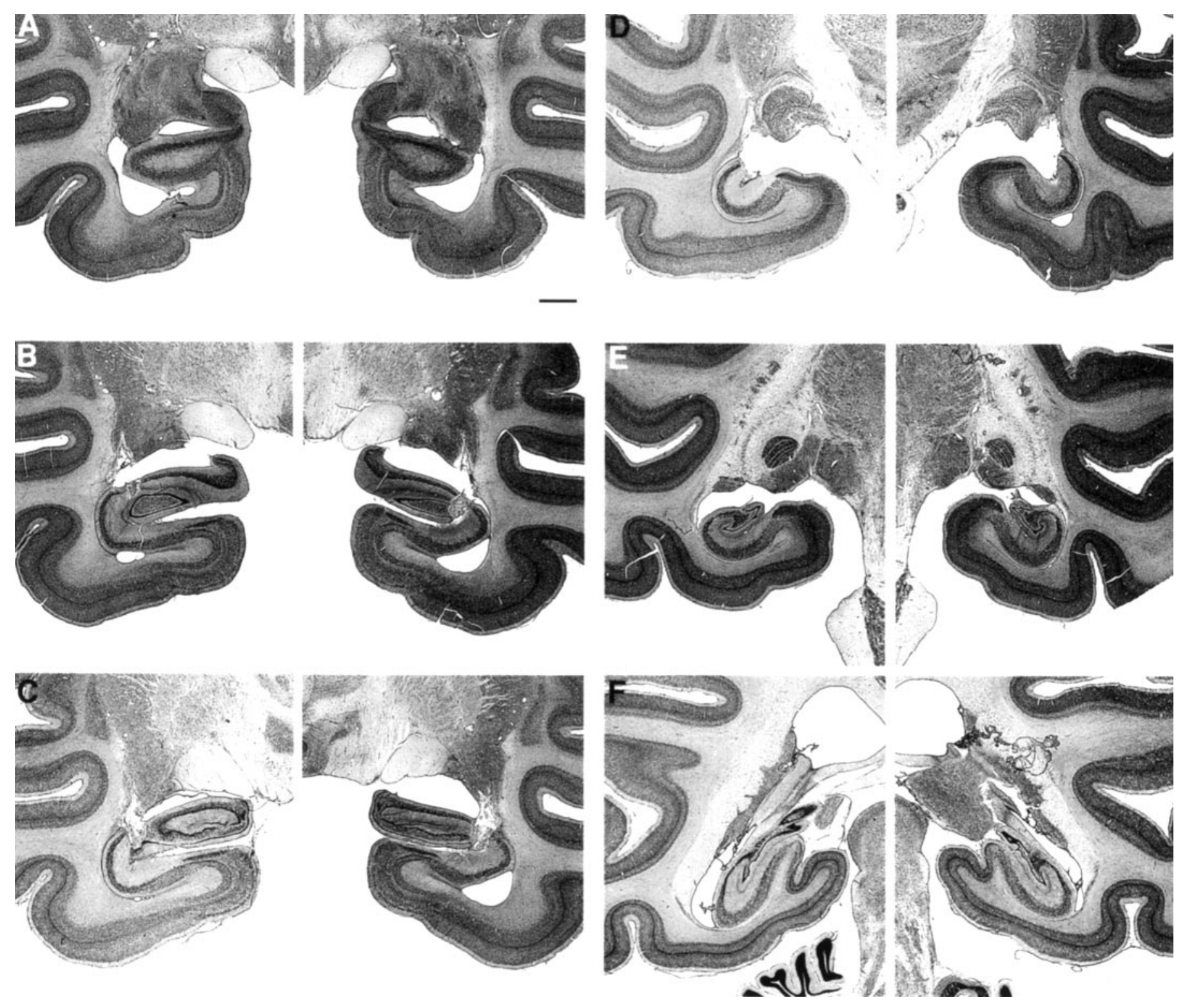

Figure 6. Photomicrographs of thionin-stained sections through the left and right temporal lobe of monkey RF2-5, whose lesion approximated the intended lesion. The sections are arranged from rostral $(A)$ to caudal $(F)$ and correspond to the line drawings in Figure 5. Scale bar (shown in $A$ for $A-F): 2 \mathrm{~mm}$.

which might then make it easier to hold novel objects in memory across the longer delays from which the performance scores for this task are derived (Bachevalier et al., 1985; Zola-Morgan and Squire, 1986; Ringo, 1988). Second, the IBO monkeys in the Murray and Mishkin (1998) study were operated on in two stages separated by at least 2 weeks. A unilateral lesion was made in the first stage, and the lesion on the other side was made in the second stage. Two-stage surgery sometimes results in less functional impairment than one-stage surgery (Finger, 1978; Finger and Stein, 1982). Although the mechanisms underlying this effect are poorly understood, the effect has been reported in the case of hippocampal lesions (Stein et al., 1969; Isseroff et al., 1976). Indeed, in some cases, deficits associated with one-stage hippocampal lesions were absent altogether after two-stage surgery (Stein et al., 1969).

In the present study, the extent of damage to the hippocampal region did not correlate with the severity of recognition memory impairment. This finding appears to differ from the finding with rodents that the volume of hippocampal lesions correlated with the degree of impairment (Moser et al., 1993). There are a number of differences between the present study in monkeys and the studies in rodents. First, different tasks were used in the two studies (visual recognition memory tasks vs the water maze). Second, in the work with rats, a large number of animals were used to demonstrate a significant correlation [84 rats were used in the analyses performed by Moser et al. (1993)]. In the present study, data for 22 animals were available. To establish a correlation between extent of damage to the hippocampus and performance on recognition memory tasks, it might be necessary to study a larger numbers of animals and also to design the study for this specific purpose.

Finally, it is possible that a correlation between extent of hippocampal damage and recognition memory performance would emerge only when some animals are available with quite 


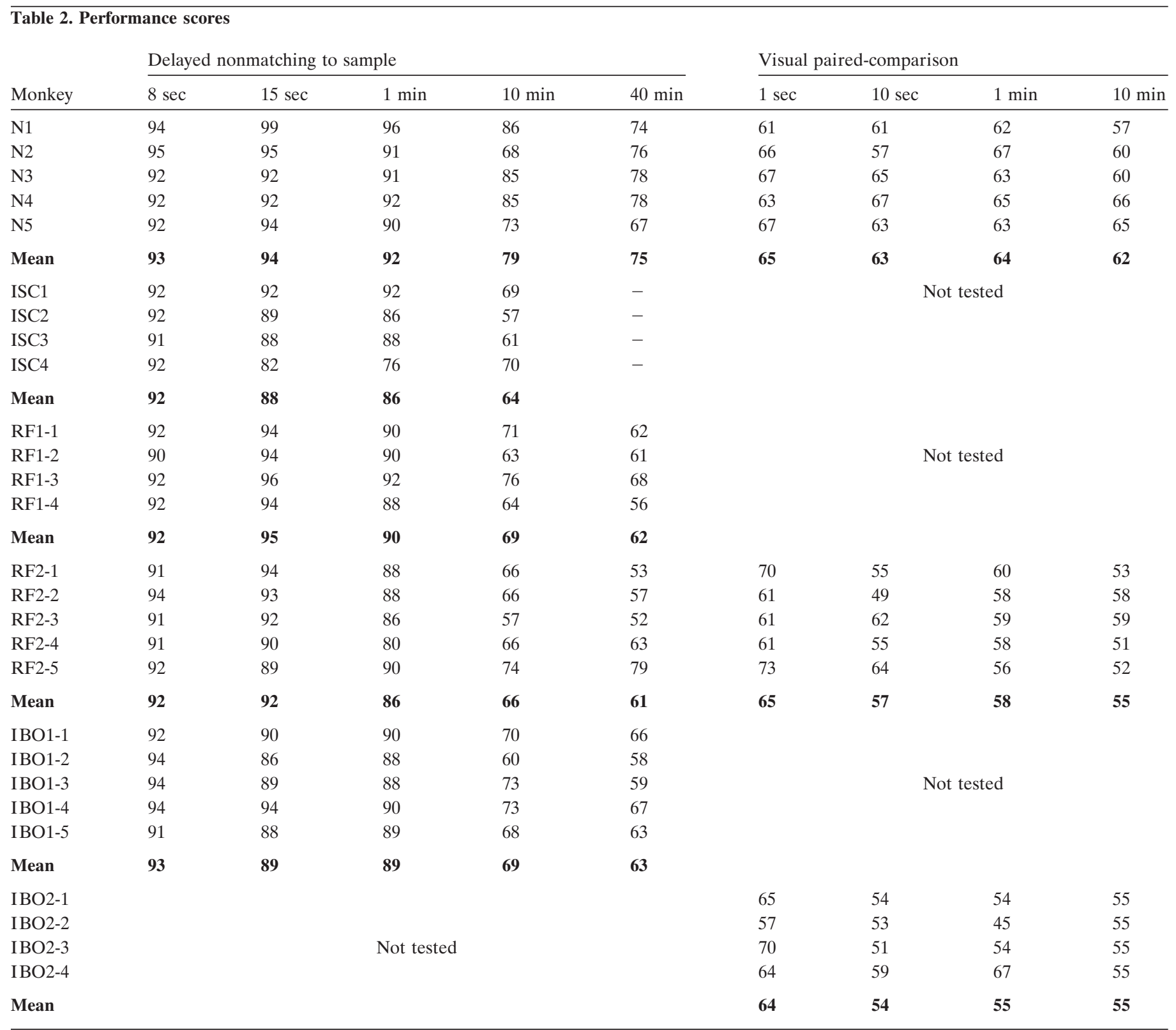

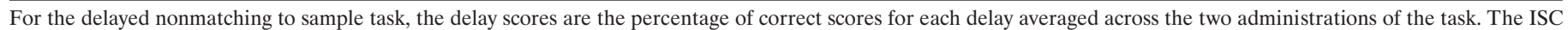

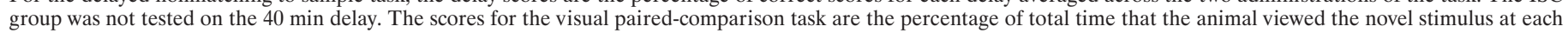
delay. N, Unoperated control monkeys; other abbreviations are as in Table 1.

small amounts of damage. In the study by Moser et al. (1993), the correlation between dorsal hippocampal lesions and water maze performance depended on the data from rats with $<20 \%$ damage to the hippocampus. Damage in excess of $20 \%$ did not increase the deficit (Moser et al., 1993, their Fig. 6). In our study, perhaps a correlation between lesion size and performance did not emerge because only three monkeys had damage that involved $<23 \%$ of the hippocampal region (Table 1). Thus, one possibility is that a lesion of at least $20 \%$ of the hippocampal region is needed to impair memory and that larger lesions do not increase the deficit. In the case of rats learning the water maze, the dorsal hippocampus is more important than the ventral hippocampus, and ventral lesions affected learning only when nearly half of the hippocampus was removed (Moser et al., 1993). The relationship between lesion size and behavioral impairment may vary according to the specific task (and kind of memory function) under study. It is not known whether visual recognition memory capacity depends more on one portion of the hippocampus than another or whether it depends equivalently on the entire hippocampal region.

The conclusion that the hippocampal region is essential for normal recognition memory is entirely consistent with many current ideas about the role of the hippocampus in declarative memory (Sutherland and Rudy, 1989; Squire, 1992a,b; Eichenbaum, 1997; Eichenbaum et al., 1999). Thus, it is often suggested that the hippocampal region and the medial temporal lobe system to which it belongs is essential for the normal acquisition of information about relationships, combinations, and conjunctions among and between stimuli. Furthermore, the anatomy of this system is consistent with the idea that the hippocampus extends and combines the functions performed by the adjacent perirhinal and parahippocampal cortices, which are positioned earlier in the 

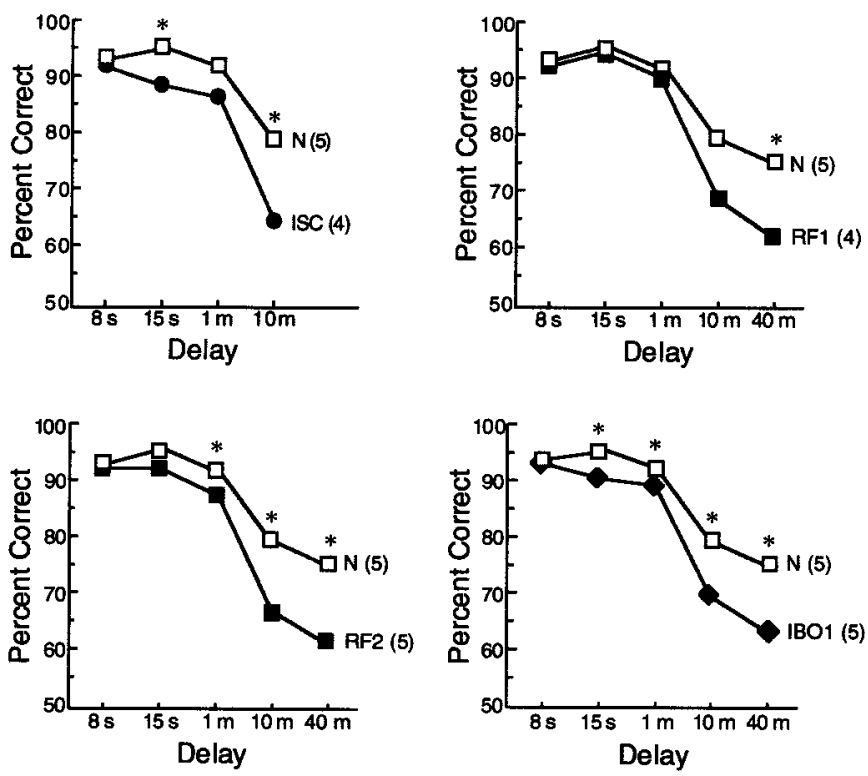

Figure 7. Delayed nonmatching to sample performance of normal monkeys $(N)$ and monkeys with lesions limited to the hippocampal region (the dentate gyrus, the cell fields of the hippocampus, and the subiculum) made by ischemia (ISC), radio frequency $(R F 1, R F 2)$, or ibotenic acid (IBO1). Asterisks indicate impaired performance of the lesion group relative to the $N$ group ( $p<0.05$ ). The $R F 1$ group performed numerically worse than the $N$ group at the 10 min delay, but the difference fell short of significance $(p<0.07)$. Parentheses indicate the number of monkeys in each group. The four lesion groups were similarly impaired.
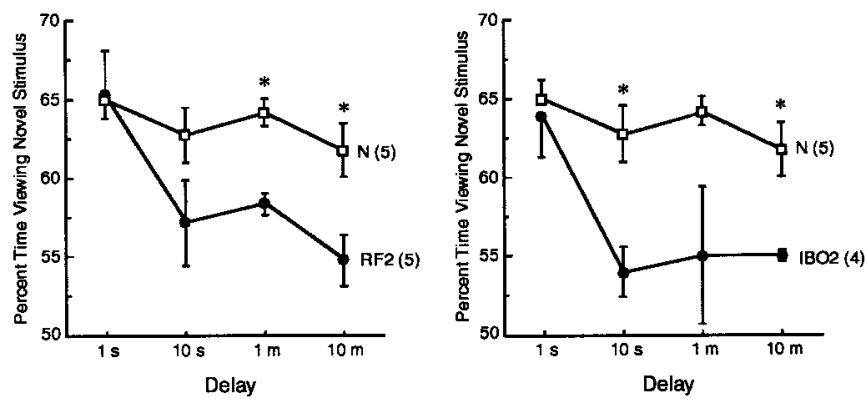

Figure 8. Visual paired-comparison performance of normal monkeys (same as in Fig. 7, $N$ ) and monkeys with radio-frequency (RF2) lesions or ibotenic acid (IBO2) lesions limited to the hippocampal region. Asterisks indicate impaired performance of the lesion group relative to the $N$ group. Parentheses indicate the number of monkeys in each group. Brackets indicate SEM. The two lesion groups were similarly impaired.

information-processing hierarchy. Recognition memory tests ask whether an item that has recently been presented subsequently appears familiar. The recognition (or familiarity) decision requires that the stimulus presented in the retention test be identified as what was presented during learning. At the time of learning a link must therefore be made between the to-beremembered stimulus and its context or between the stimulus and the animal's interaction with it. It is this process of forming associations and the ability to retain relational information across time that many have supposed is at the heart of declarative memory and in turn is the function of the hippocampal region in both humans and animals.
Delayed Nonmatching to Sample

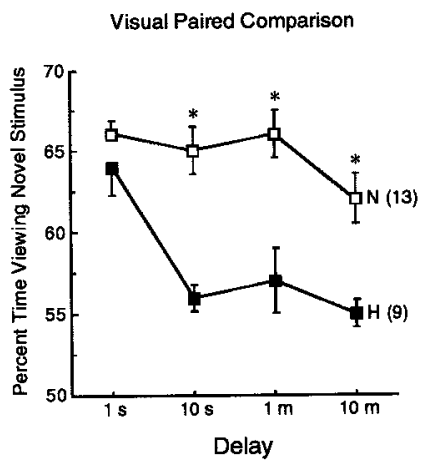

Figure 9. Performance on the delayed nonmatching to sample task (left panel) and on the visual paired-comparison task (right panel) by all the monkeys in the current study with lesions limited to the hippocampal region. All the normal monkeys $(N)$ that have been tested previously in our laboratory on these two tasks are also included. Asterisks indicate impaired performance of the lesion group relative to the $N$ group. Parentheses indicate the number of monkeys in each group. ${ }^{\text {a The ISC }}$ monkeys were not tested at the $40 \mathrm{sec}$ delay so that the scores of 14 monkeys contributed to this data point.

\section{REFERENCES}

Aggleton JP, Brown MW (1999) Episodic memory, amnesia, and the hippocampal-anterior thalamic axis. Behav Brain Sci 22:425-444.

Aggleton JP, Shaw C (1996) Amnesia and recognition memory: a reanalysis of psychometric data. Neuropsychologia 34:51-62.

Alvarez P, Zola-Morgan S, Squire LR (1995) Damage limited to the hippocampal region produces long-lasting memory impairment in monkeys. J Neurosci 15:3796-3807.

Alvarez-Royo P, Clower RP, Zola-Morgan S, Squire LR (1991) Stereotaxic lesions of the hippocampus in monkeys: determination of surgical coordinates and analysis of lesions using magnetic resonance imaging. J Neurosci Methods 38:223-232.

Bachevalier J, Meunier M (1996) Cerebral ischemia: are the memory deficits associated with hippocampal cell loss? Hippocampus 6:553-560.

Bachevalier J, Saunders RC, Mishkin M (1985) Visual recognition in monkeys: effects of transection of fornix. Exp Brain Res 57:547-553.

Bachevalier J, Brickson M, Hagger C (1993) Limbic-dependent recognition memory in monkeys develops early in infancy. NeuroReport 4:77-80.

Beason-Held LL, Rosene DL, Killiany RJ, Moss MB (1999) Hippocampal formation lesions produce memory impairment in the rhesus monkey. Hippocampus 9:562-574.

Buffalo EA, Reber PJ, Squire LR (1998) The human perirhinal cortex and recognition memory. Hippocampus 8:330-339.

Eichenbaum H (1997) Declarative memory: insights from cognitive psychology. Annu Rev Psychol 48:547-572.

Eichenbaum H, Otto T, Cohen, NJ (1994) Two functional components of the hippocampal memory system. Behav Brain Sci 17:449-518.

Eichenbaum H, Dudchenko P, Wood E, Shapiro M, Tanila H (1999) The hippocampus, memory, and place cells: is it spatial memory or a memory space? Neuron 23:209-226.

Finger S (1978) Recovery from brain damage: research and theory. New York: Plenum.

Finger S, Stein DG (1982) Brain damage and recovery: research and clinical perspectives. New York: Academic.

Gellerman LW (1933) Chance orders of alternating stimuli in visual discrimination experiments. J Gen Psychol 42:207-208.

Harlow H, Bromer JA (1938) A test-apparatus for monkeys. Psychol Rev 19:434-438.

Isseroff A, Leveton L, Freeman G, Lewis ME, Stein DG (1976) Differences in the behavioral effects of single-stage and serial lesions of the hippocampus. Exp Neurol 53:339-354.

Jarrard LE (1989) On the use of ibotenic acid to lesion selectively different components of the hippocampal formation. J Neurosci Methods 29:251-259.

Manns JR, Squire LR (1999) Impaired recognition memory on the Doors and People Test after damage limited to the hippocampal region. Hippocampus 9:495-499. 
McKee RD, Squire LR (1993) On the development of declarative memory. J Exp Psychol 19:397-404.

Mishkin M, Delacour J (1975) An analysis of short-term visual memory in the monkey. J Exp Psychol 1:326-334.

Moser E, Moser M (1998) Functional differentiation in the hippocampus. Hippocampus 8:608-619.

Moser E, Moser M, Andersen P (1993) Spatial learning impairment parallels the magnitude of dorsal hippocampal lesions, but is hardly present following ventral lesions. J Neurosci 13:3916-3925.

Moser MB, Moser EI, Forrest E, Anderson P, Morris RG (1995) Spatial learning with a minislab in the dorsal hippocampus. Proc Natl Acad Sci USA 92:9697-9701.

Murray EA (1990) Representational memory in nonhuman primates. In: Neurobiology of comparative cognition (Kesner RP, Olton DS, eds), pp 127-155. Hillsdale, NJ: Lawrence Erlbaum Associates.

Murray EA, Mishkin M (1998) Object recognition and location memory in monkeys with excitotoxic lesions of the amygdala and hippocampus. J Neurosci 18:6568-6582.

Nadel L (1995) The role of the hippocampus in declarative memory: a comment on Zola-Morgan, Squire, and Ramus (1994). Hippocampus 5:232-234.

Nunn J, Hodges H (1994) Cognitive deficits induced by global cerebral ischaemia: relationship to brain damage and reversal by transplants. Behav Brain Res 65:1-31.

Reed JM, Squire LR (1997) Impaired recognition memory in patients with lesions limited to the hippocampal formation. Behav Neurosci 111:667-675

Rempel-Clower N, Zola SM, Squire LR (1996) Three cases of enduring memory impairment following bilateral damage limited to the hippocampal formation. J Neurosci 16:5233-5255.

Ringo JL (1988) Seemingly discrepant data from hippocampectomized macaques are reconciled by detectability analysis. Behav Neurosci 102:173-177.

Squire LR (1992a) Declarative and nondeclarative memory: multiple brain systems supporting learning and memory. J Cogn Neurosci 4:232-243.

Squire LR (1992b) Memory and the hippocampus: a synthesis from findings with rats, monkeys and humans. Psychol Rev 99:195-231.

Squire LR, Zola SM (1996) Ischemic brain damage and memory impairment: a commentary. Hippocampus 6:546-552.

Squire LR, Zola-Morgan S, Chen K (1988) Human amnesia and animal models of amnesia: performance of amnesic patients on tests designed for the monkey. Behav Neurosci 11:210-221.

Stein DG, Rosen JJ, Graziadei J, Mishkin D, Brink JJ (1969) Central nervous system: recovery of function. Science 166:528-530.

Sutherland RW, Rudy JW (1989) Configural association theory: the role of the hippocampal formation in learning, memory and amnesia. Psychobiology 17:129-144.

Szabo J, Cowan, WM (1984) A stereotaxic atlas of the brain of the cynomolgus monkey (Macaca fascicularis). J Comp Neurol 222:265-300.

Teng E, Squire LR, Zola SM (1998) Effects of removal from the testing apparatus on delayed nonmatching to sample performance in monkeys with lesions of the hippocampal region. Soc Neurosci Abstr 24:17.

Vargha-Khadem F, Gadian DG, Watkins KE, Connelly A, Van Paesschen W, Mishkin M (1997) Differential effects of early hippocampal pathology on episodic and semantic memory. Science 277:376-380.

Vargha-Khadem F, Watkins KE, Baddeley AD, Mishkin M (1998) Dissociation between recognition and recall after early hippocampal damage. Soc Neurosci Abstr 24:1523.

Wang J, Aigner T, Mishkin M (1990) Effects of neostriatal lesions on visual habit formation in rhesus monkeys. Soc Neurosci Abstr 16:617.

Zola SM, Squire LR (2000) Memory and brain systems. In: Oxford handbook of memory (Tulving E, Craik F, eds). New York: Oxford UP, in press.

Zola SM, Teng E, Clark RE, Stefanacci L, Buffalo EA, Squire LR (1998) Impaired recognition memory and simple discrimination learning in monkeys following lesions limited to the hippocampal region made by radio frequency, ischemia, or ibotenic acid. Soc Neurosci Abstr 24:17.

Zola-Morgan S, Squire LR (1986) Memory impairment in monkeys following lesions of the hippocampus. Behav Neurosci 100:165-170.

Zola-Morgan S, Squire LR (1993) Neuroanatomy of memory. Annu Rev Neurosci 16:547-563.

Zola-Morgan S, Squire LR, Amaral DG (1986) Human amnesia and the medial temporal region: enduring memory impairment following a bilateral lesion limited to field CA1 of the hippocampus. J Neurosci 6:2950-2967.

Zola-Morgan S, Squire LR, Rempel NL, Clower RP, Amaral DG (1992) Enduring memory impairment in monkeys after ischemic damage to the hippocampus. J Neurosci 9:4355-4370.

Zola-Morgan S, Squire LR, Ramus SJ (1994) Severity of memory impairment in monkeys as a function of locus and extent of damage within the medial temporal lobe memory system. Hippocampus 4:483-495. 